\title{
Mechanical properties and constitutive analyses of basalt fiber crumb rubber mortars in soft rock roadways
}

\author{
Qiao Wei-Guo ${ }^{1}$, Vladimir Pershin ${ }^{2, *}$ Qin Jun-Ling ${ }^{1}$, and Zhang Shuai ${ }^{1}$ \\ ${ }^{1}$ Shandong University of Science and Technology, Qingdao 266590, China \\ ${ }^{2}$ T. F. Gorbachev Kuzbass State Technical University, Kemerovo 650026, Russia
}

\begin{abstract}
Uniaxial compressive stress-strain curves of several groups of basalt fiber crumb rubber mortars (BF-CRM) with different contents are tested. By comparing with the GZH model, the improved values of the parameters of the BF-CRM constitutive model based on the GZH model are obtained. Then, taking the supporting scheme of main substation of the mine as the background, using FLAC3D to simulate the roadway support, using BF-CRM to replace the ordinary mortars in the original support, and combining with the triaxial compression test of different confining pressure, the application effect of BF-CRM in roadways support is studied and analyzed.
\end{abstract}

\section{Introduction}

The addition of crumb rubber into engineering materials such as cement mortar not only improves the ductility, durability and impact resistance of cement mortar, but also promotes the reuse of waste rubber ${ }^{[1,2]}$. Basalt fiber is a kind of silicate fiber processed from volcanic rock ore, which has the advantages of high tensile strength, high temperature resistance, corrosion resistance and stable chemical properties ${ }^{[3,4]}$. Crumb rubber or basalt fibers are incorporated into traditional building materials to form new composite building materials, which have been widely introduced into pavement engineering, protection engineering, high-speed railway and underground space engineering ${ }^{[5,6]}$.

In recent years, scholars worldwide have conducted lots of research on composite materials based cement mortars. Yan Handong ${ }^{[7]}$ Studied the influence of rubber powder on fresh mortar and hardened mortar. The results showed that the workability of fresh mortar can be improved, the deformability of hardened mortar can be improved, and the early strength and frost resistance of hardened mortar can be improved by adding appropriate amount of rubber powder. Long Guangcheng ${ }^{[8,9]}$ studied the mechanical properties of cement concrete with waste rubber particles of different sizes. Yong $\mathrm{Yu}^{[10]}$ found that the consistency and initial density of cement mortar decreased with the increase of rubber

\footnotetext{
* Corresponding author: pvv.spssh@,kuzstu.ru
} 
particle size, and the porosity increased with the decrease of rubber particle size. Xiong Rui $^{[11]}$ studied the reinforcing effect of basalt fibers and other fibers on cement matrix materials, which showed that the compressive and flexural strength of cement matrix could be effectively improved by the addition of fibers, especially the basalt fibers impregnated. $\mathrm{Li}$ Weimin ${ }^{[12]}$ studied the effect of basalt fibers on the dynamic mechanical properties of concrete under impact loading. It was found that when the content of basalt fibers was $0.1 \%$, the effect of basalt fibers on strengthening and toughening was the best. Luigi Fenu ${ }^{[13]}$ conducted static and dynamic tests to study the effects of glass fiber and basalt fiber on the tensile strength, flexural strength and energy absorption capacity of cement mortar respectively. The results showed that both fibers can increase the energy absorption capacity of cement mortar. M. L. Santarelli ${ }^{[14]}$ studied the physical and mechanical properties and micro structures of three kinds of hydrated lime mortar with different compositions by basalt fibers. It was found that basalt fibers can delay the cracking of mortar, improve the compressive strength.

In this paper, BF-CRM specimens with different basalt fiber content and rubber particle content were designed, and conducted the uniaxial compression tests. The stress-strain curves obtained by tests were compared with the model of $\mathrm{GZH}^{[15]}$, and the recommended values of BF-CRM constitutive parameters were proposed by fitting the test data. Then, based on the tunnel support scheme of a mine main substation, using FLAC3D and combined with BF-CRM triaxial compression tests under $5 \mathrm{MPa}$ and $8 \mathrm{MPa}$ confining pressure, the application effect of BF-CRM in high stress soft rock roadway support was studied and analyzed.

\section{Experimental study of physical mechanics}

\subsection{Materials and mixing ratio}

The physical and mechanical properties of the short-cut basalt fibers produced by Anjie in Zhejiang province are shown in Table 1 . The bulk density of river sand is $1440 \mathrm{~kg} / \mathrm{m}^{3}$, and the fineness modulus is 2.4. The particle size of the rubber is $6-8 \mathrm{~mm}$ and the apparent density is $1200 \mathrm{~g} / \mathrm{cm}^{3}$. Before preparing the test specimens, the rubber particles were first placed in $10 \% \mathrm{NaOH}$ solution and immersed for 30 minutes. Additives mainly include water reducer and binders.

The mixing ratio of basalt fiber crumb rubber mortar are as follows. The ratio of water to cement is 0.35 and the ratio sand to cement is 1.5 . The proportion of rubber particles is $0 \%, 5 \%, 10 \%, 20 \%, 30 \%$, which is expressed by $\mathrm{A}, \mathrm{B}, \mathrm{C}, \mathrm{D}$ and $\mathrm{E}$, respectively. The content of basalt particles is $0 \%, 0.4 \%, 0.8 \%$ and $1.2 \%$. For example, B- $0.4 \%$ means that the rubber particle content of BF-CRM is $5 \%$ of the total mass of BF-CRM and the basalt fiber content is $0.4 \%$ of the total mass of BF-CRM.

Table 1. Physical and mechanical properties of basalt fiber

\begin{tabular}{|c|c|c|c|c|c|}
\hline $\begin{array}{c}\text { Length/m } \\
\mathbf{m} \\
\end{array}$ & $\begin{array}{c}\text { Diameter/ } \mu \\
\mathbf{m} \\
\end{array}$ & Density $/\left(\mathrm{kg} / \mathrm{m}^{3}\right)$ & $\begin{array}{c}\text { Tensile } \\
\text { strength/MPa }\end{array}$ & $\begin{array}{c}\text { Elastic } \\
\text { modulus/GPa }\end{array}$ & Elongation/\% \\
\hline 20 & 15 & 2650 & $4100-4840$ & $90-110$ & $2.4-3.0$ \\
\hline
\end{tabular}




\section{Results and discussion}

Twenty groups of BF-CRM specimens were designed in this experiment. Each group contains four specimens. Three specimens were used for uniaxial compression test and one was reserved, the size of the specimens is a $\varphi 50 \mathrm{~mm} \times 100 \mathrm{~mm}$ cylinder.

Under uniaxial compression, compared with the same basalt fiber content, the compression of the mortar increases with the increase of rubber particle content. When the rubber particle content is less than $20 \%$, the compression increases more smoothly, and when the rubber content is more than $20 \%$, the compression increases faster. The increase of basalt fiber content has less obvious effect on the compression of BF-CRM .

The failure modes of each group of specimens after uniaxial compression test are showed in FIG. 1. For group A specimens without rubber particles, during the loading process, some small cracks gradually appear in the middle of the specimen, and the surface has obvious spalling phenomenon. After reaching the peak stress, the width of cracks increases continuously, and the bearing capacity of the test block decreases rapidly. During loading of group B, C and D specimens, the rate growth of crack gradually slows down with the increase of basalt fiber content, and the addition of fiber delays the appearance and penetration of crack. In group E, there were no obvious cracks in the loading process, and the specimens could still maintain good integrity until the end of loading.

By averaging the results of 3 tests in each group, the stress-strain curves of each group
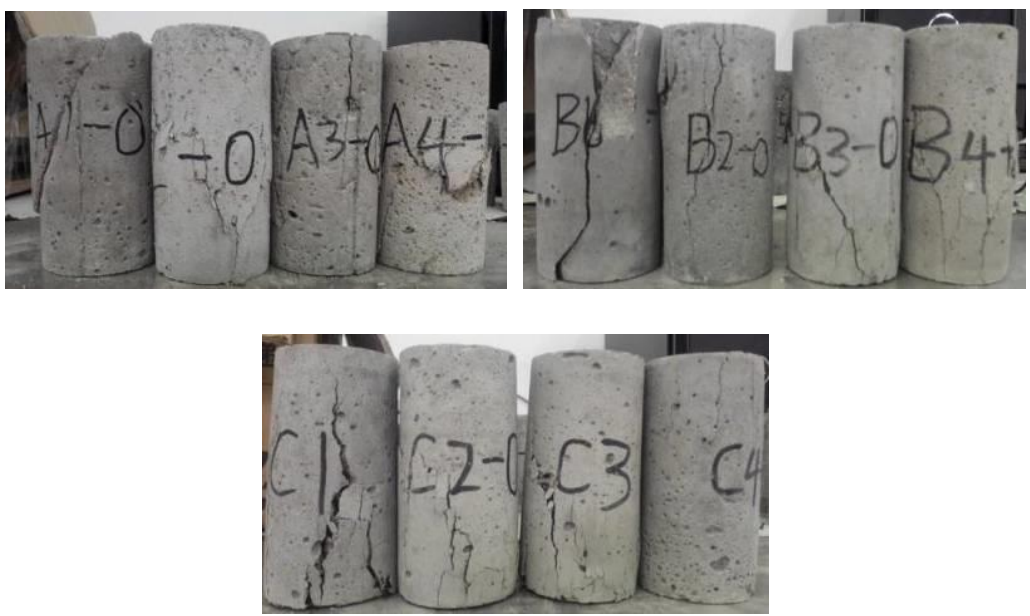

A group

B group

C group

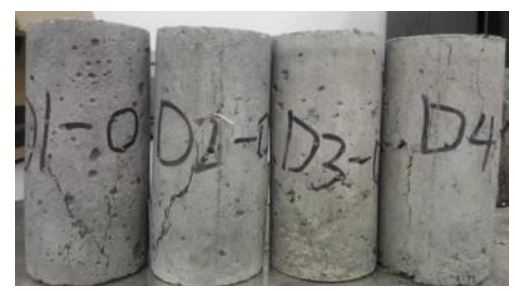

D group

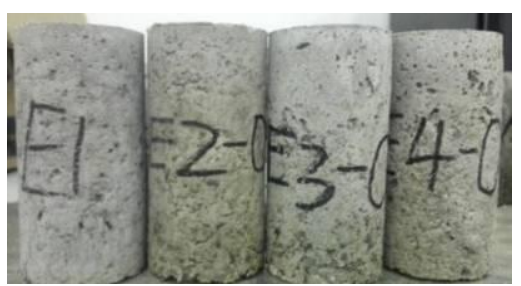

E group

Fig. 2. Uniaxial failure modes of basalt fiber rubber particles mortar

are shown in FIG. 2. It can be seen that the peak strength of the test decreases gradually 
with the increase of rubber particle content. When the rubber particle content is $30 \%$, the peak strength is only about $1.5 \mathrm{MPa}$. After reaching the peak strength, the ductility of the specimens increase gradually; moreover, with the increase of rubber particle content, the compaction stage of the tests increases significantly during the loading process. With the increase of fiber content, the stress-strain curve of the tests moves up and the peak stress increases. When the fiber content is $0.4 \%$, the effect of increasing the peak stress is the best.

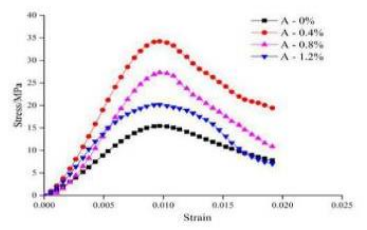

A group

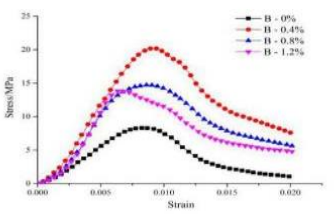

B group

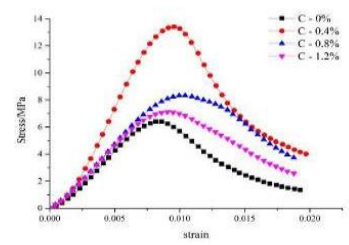

C group

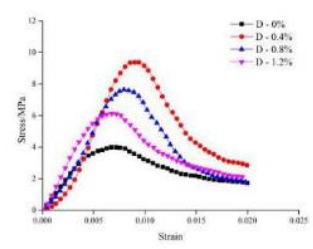

D group

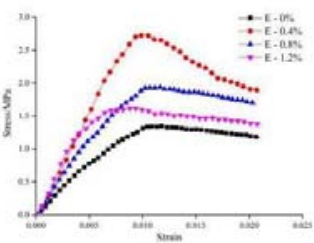

E group

Fig. 2. Experimental stress-strain curves of BF-CRM under uniaxial compression

Uniaxial Compression Constitutive Model

In order to analyze the mechanical properties of BF-CRM under uniaxial compression, the stress-strain curves obtained from the tests are compared and evaluated with the GZH model. Based on the evaluation results, the revised recommended values for BF-CRM constitutive parameters are given.

The GZH model is the piecewise formula for describing the stress-strain curve of ordinary concrete under uniaxial compression, which is proposed by Guo Zhenhai based on the results from tests, as follows:

$$
\begin{gathered}
y=a x+(3-2 a) x^{2}+(a-2) x^{3}, x<1 \\
y=\frac{x}{b(x-1)^{2}+x}, x \geq 1 \\
x=\frac{\varepsilon}{\varepsilon_{c}}, y=\frac{f}{\sigma_{c}}
\end{gathered}
$$

In the formula $\mathrm{a}$ and $\mathrm{b}$ are the parameters of constitutive model, $\sigma 、 \varepsilon$ is stress and strain respectively, $f_{c} 、 \varepsilon_{c}$ is peak stress and peak strain respectively.

Comparison of stress- strain curve and gzh model 
The experimental stress-strain curves of BF-CRM are compared with the GZH model, as shown in FIG. 3. In the rising section of stress-strain curve, the GZH model is obviously different from the test curve. Except for the curve of A- $0 \%$ and $\mathrm{C}-0 \%$, the GZH model obviously overestimates the bearing capacity of other test groups, and which is related to the amount of rubber particles and basalt fiber. In the descending section of test group curve, especially with the increase of rubber particle content, the change of ductility of test block can be well reflected. But the stress calculated by GZH model is generally lower than the value of tests, and when the peak strength of the test is lower than $10 \mathrm{MPa}$, the curve of GZH model deviates from the test curve. Therefore, it is not appropriate to directly use GZH model to analyze and calculate BF-CRM specimens.

MODIFICATION OF BF-CRM CONSTITUTIVE MODEL

Unlike ordinary cement mortar, BF-CRM involves many factors such as rubber particle content, particle size, basalt fiber content and so on. Therefore, on the basis of the stress-strain test data and the GZH model, the fitting values of the constitutive parameters a and $b$ are carried out to obtain the modified GZH model for BF-CRM.

The stress-strain curve of the tests were compared with the modified GZH model curve as shown in FIG. 4. The modified GZH model agrees well with the experimental curve, especially in the descending section of the curve, which reflects the ductility of BF-CRM. It is worth noting that in the compression stage of the stress-strain curve, the modified GZH model will slightly overestimate the strength of the material.The values of fitting parameters a and $\mathrm{b}$ based on GZH model are shown in TABLE 2.

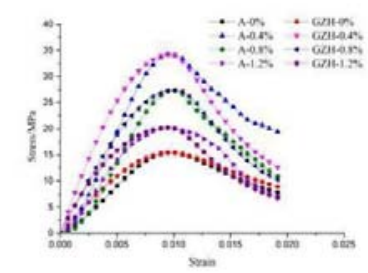

A group

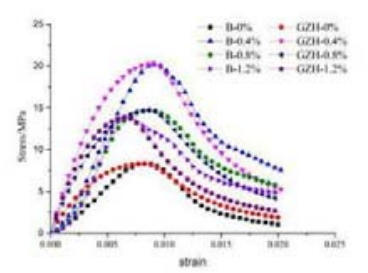

B group

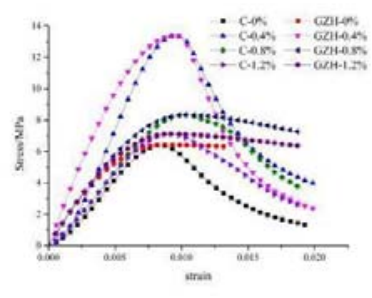

C group

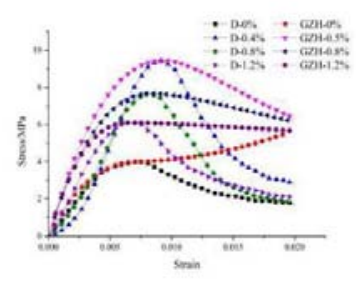

D group

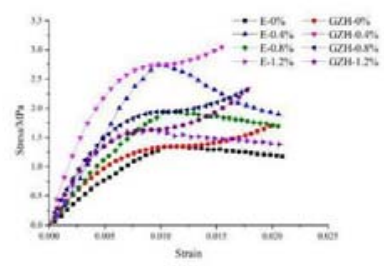

E group

Fig. 3. Fitting results of the curve of BF-CRM based on GZH model 


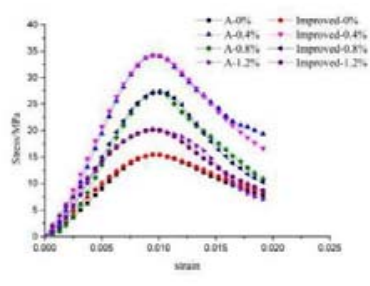

A group

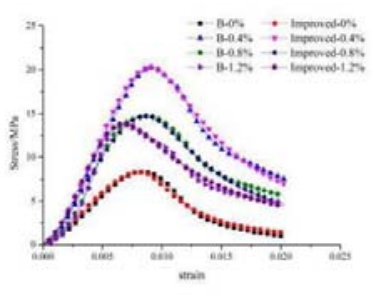

B group

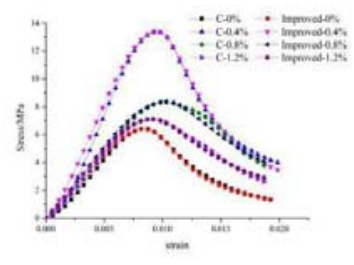

C group

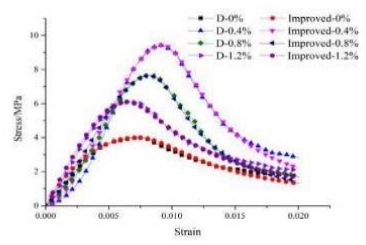

D group

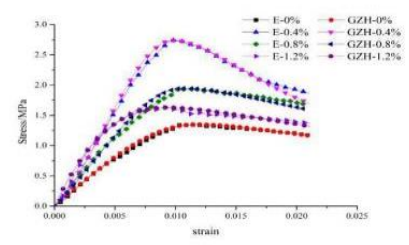

E group

Fig. 4. Fitting results of the curve of BF-CRM based on the Modified GZH model

Numerical Simulation of BF-CRM in Roadway Support

FLAC3D was used to simulate and analyze the roadway support based on the geological conditions and support scheme of a mine main substation channel. In order to explore the application effect of basalt fibre rubber granular mortar in soft rock roadway support engineering, $400 \mathrm{~mm}$ ordinary mortar (plan A) is replaced by $200 \mathrm{~mm}$ basalt fibre rubber granular mortar (plan B) in the original roadway support scheme. The BF-CRM layer was based on the physical and mechanical parameters obtained from the B- $0.4 \%$ group test.

DISPLACEMENT EVOTION CHARACTERISTICS OF ROADWAY

Analysing plan A, after $6 \mathrm{~m}$ of roadway excavation, adopting plan A to support. The maximum vertical displacement of floor at $\mathrm{y}=0$ is $437 \mathrm{~mm}$, and the maximum displacement

Table 2. Fitting parameters a and b based on GZH model

\begin{tabular}{|c|c|c|c|c|c|}
\hline Type & Fitting & Fitting & Type & Fitting & Fitting \\
\hline A - $0 \%$ & 1.007 & 1.718 & D - 0\% & 2.350 & 1.903 \\
\hline A- $0.4 \%$ & 0.641 & 2.181 & D- $0.4 \%$ & 0.132 & 5.230 \\
\hline A- $0.8 \%$ & 0.158 & 4.019 & D- $0.8 \%$ & 0.654 & 5.227 \\
\hline A- $1.2 \%$ & 1.447 & 2.733 & D- $1.2 \%$ & 1.424 & 1.842 \\
\hline B - $0 \%$ & 0.696 & 5.743 & E- $\% \%$ & 1.483 & 0.446 \\
\hline B- $0.4 \%$ & 0.347 & 2.745 & E- $0.4 \%$ & 0.666 & 1.013 \\
\hline B- $0.8 \%$ & 0.666 & 2.776 & E- $0.8 \%$ & 1.076 & 0.457 \\
\hline B- $1.2 \%$ & 0.327 & 1.587 & E- $1.2 \%$ & 2.380 & 0.318 \\
\hline
\end{tabular}




\begin{tabular}{|l|l|l|l|l|l|}
\hline $\mathrm{C}-0 \%$ & 0.770 & 5.530 & & & \\
\hline $\mathrm{C}-0.4 \%$ & 0.632 & 5.129 & & & \\
\hline $\mathrm{C}-0.8 \%$ & 1.046 & 2.852 & & & \\
\hline $\mathrm{C}-1.2 \%$ & 1.061 & 2.651 & & & \\
\hline
\end{tabular}

of floor at $y=5$ is $400 \mathrm{~mm}$. For the unexplored roadway, the vertical displacement is also changed at $y=10$ due to the influence of excavation, the maximum change is about $12 \mathrm{~mm}$.

Adopting plan B to support. It can be seen that the maximum vertical displacement of the floor at $\mathrm{y}=0$, i.e. the floor heave is only $35 \mathrm{~mm}$; at $\mathrm{y}=5$, the maximum displacement of the floor is $70 \mathrm{~mm}$; for the unexplored roadway part, at $\mathrm{y}=10$, the vertical displacement also varies with the maximum change of about $6 \mathrm{~mm}$ under the influence of excavation.

After replacing ordinary cement mortar with BF-CRM, the floor heave at $y=0$ is only $1 / 10$ of that at the original supporting scheme, and the floor heave at $y=6$ is also significantly reduced. The whole supporting structure better limits the floor heave deformation.

\section{MECHANISM ANALYSIS OF TWO SUPPORT SCHEMES}

According to the proportion of B- $0.4 \%$, making the BF-CRM specimens and the common cement mortar (CM) specimens with the same water cement ratio, and the triaxial compression tests were carried out under confining pressure of $5 \mathrm{MPa}$ and $8 \mathrm{MPa}$, respectively. The stress-strain curves of BF-CRM and $\mathrm{CM}$ specimens under different confining pressures were shown in FIG. 5.

It can be known that the stress-strain curve of BF-CRM is located above the stress-strain curve of ordinary CM as a whole, and the strain energy of BF-CRM under the same confining pressure is obviously higher than that of ordinary $\mathrm{CM}$, and the greater the confining pressure, the more significant it is.

Plan B is superior to plan A in controlling displacement and deformation of floor. The main reason is that BF-CRM has better compressibility. In the enclosed space between common CM layer and surrounding rock, with the deformation of surrounding rock, BF-CRM layer is squeezed, because of its large compressibility, along with surrounding rock, it can gradually absorb the energy released by confining pressure deformation, thus playing the role of buffer energy absorbing layer.

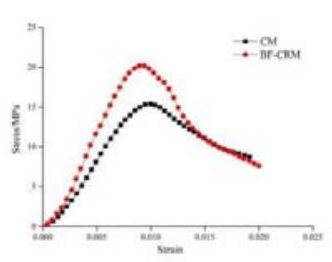

$0 \mathrm{MPa}$

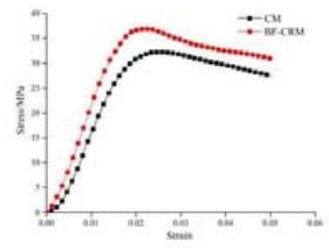

$5 \mathrm{MPa}$

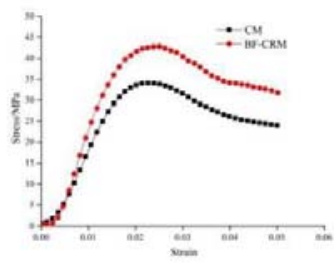

$8 \mathrm{MPa}$

Fig. 5. Stress - Strain curve under conventional triaxial pressure

\section{Conclusions}

(1) With the increase of rubber particle content, the peak stress of BF-CRM decreases 
gradually. The uniaxial compressive strength of BF-CRM can be effectively improved by adding basalt fibers. Rubber particles can improve the compressibility of cement mortar, while basalt fibers have no significant effect on the compressibility of cement mortar, but they can effectively slow down the expansion of BF-CRM cracks.

(2) By fitting the stress-strain curve obtained from the test with the GZH model, the revised values of the constitutive parameters of the GZH model are obtained. The revised GZH model is in good agreement with the stress-strain curve of the test, and can be used as a reference model for the analysis and design of BF-CRM.

(3) Taking the tunnel support scheme of a mine main substation as the background and using FLAC3D to simulate the roadway support, and combined with triaxial compression tests of $5 \mathrm{MPa}$ and $8 \mathrm{MPa}$ confining pressure, the analysis shows that compared with the original support scheme, BF-CRM layer has the function of cushioning energy absorbing layer. In soft rock roadway support, it has good effect on controlling the displacement and deformation of floor, reducing the stress concentration.

\section{Reference}

1. L. J. Hunag, H. Y. Wang, S.-Y. Wang, Constr. Build. Mater, 96, 353 (2015)

2. Y. Yu, H. Zhu, Materials, 9:7, 527, (2016)

3. K. Bisht, P.V. Ramana, Constr. Build. Mater, 155, 811 (2017)

4. Y. X. Yang, Q. R. Yue, Industrial Construction, 37:6, 1 (2007)

5. C. Colombo, L. Vergani, M. Burman, Compos Struct, 94:3, 1165 (2012)

6. V. Fiore, T. Scalici, G. Di Bella, et al, Composites Part B, 74, 74 (2015)

7. H.D. Yan, X.f. Chen, X.X. Ma, et al, China Civil Engineering Journal, 41:12, 55 (2008)

8. G.C. Long, N. Li, Y.H. Xue, et al, J Chin Ceram Soc, 44:8, 1081 (2016)

9. H.B. Zhang, X.M. Guan, M.F. Gou, et al, Bulletin of the Chinese Ceramic Society, 31:4, 931 (2012)

10. Y. Yu, H. Zhu, Materials, 9:7, 527 (2016)

11. R. Xiong, Z.G. Ren, Z.A. Lu, et al, Journal of Wuhan university of technology, 35:7, 109 (2013)

12. W.M. Li, J.Y. Xu, J Chin Ceram Soc, 36:4, 476 (2008)

13. Luigi Fenu, Daniele Forni, Ezio Cadoni, Composites Part B, 92, 142 (2016)

14. M. L. Santarelli, F. Sbardella, BARDELLA, M. Zuena, et al, Materials and Design, 63, 398 (2014)

15. Z.H. Guo, X.Q. Zhang, D.C. Zhang, et al, Journal of building Structures, 3:1, 3 (1982) 